\title{
Conservative Difference Scheme for Generalized Rosenau-KdV Equation
}

\author{
Yan Luo, ${ }^{1}$ Youcai Xu, ${ }^{2}$ and Minfu Feng ${ }^{2}$ \\ ${ }^{1}$ School of Mathematical Sciences, University of Electronic Science and Technology of China, Chengdu 610054, China \\ ${ }^{2}$ School of Mathematics, Sichuan University, Chengdu 610064, China \\ Correspondence should be addressed to Yan Luo; luoyan_16@126.com
}

Received 12 January 2014; Revised 9 April 2014; Accepted 22 April 2014; Published 14 May 2014

Academic Editor: Ricardo Weder

Copyright (c) 2014 Yan Luo et al. This is an open access article distributed under the Creative Commons Attribution License, which permits unrestricted use, distribution, and reproduction in any medium, provided the original work is properly cited.

A conservative Crank-Nicolson finite difference scheme for the initial-boundary value problem of generalized Rosenau-KdV equation is proposed. The difference scheme shows a discrete analogue of the main conservation law associated to the equation. On the other hand the scheme is implicit and stable with second order convergence. Numerical experiments verify the theoretical results.

\section{Introduction}

In this paper, we consider the following initial-boundary value problem of the generalized Rosenau-KdV equation:

$$
u_{t}+u_{x x x x t}+u_{x}+\left(u^{p}\right)_{x}+u_{x x x}=0
$$

with an initial condition

$$
u(x, 0)=u_{0}(x), \quad x \in\left[x_{L}, x_{R}\right],
$$

and boundary conditions

$$
\begin{aligned}
& u\left(x_{L}, t\right)=u\left(x_{R}, t\right)=0, \\
& u_{x}\left(x_{L}, t\right)=u_{x}\left(x_{R}, t\right)=0, \\
& u_{x x}\left(x_{L}, t\right)=u_{x x}\left(x_{R}, t\right)=0, \\
& t \in[0, T],
\end{aligned}
$$

where $p \geq 2$ is a integer and $u_{0}(x)$ is a known smooth function. When $p=2$, (1) is called usual Rosenau-KdV equation:

$$
u_{t}+u_{x x x x t}+u_{x}+u u_{x}+u_{x x x}=0 .
$$

Zuo [1] discussed the solitary wave solutions and periodic solutions for Rosenau-KdV equation. In [2], a conservative nonlinear finite difference scheme for an initial-boundary value problem of Rosenau-Kdv equation is considered.

In $[3,4]$ the solitary solution and invariant for generalized Rosenau-KdV equation are given. In [4] the singular 1soliton solution is derived by the ansatz method, and the adiabatic parameter dynamics of the water waves is obtained by perturbation theory. In $[5,6]$, the ansatz method is applied to obtain the topological soliton solution of the generalized Rosenau-KdV equation. The method as well as the exp-function method is also applied to extract a few more solutions to this equation. In [7], Zheng and Zhou give an average linear scheme for the generalized Rosenau-KdV equation. In this paper, we propose a conservative CrankNicolson finite difference scheme for an initial-boundary value problem of the generalized Rosenau-Kdv equation.

The initial-boundary value problem (1)-(3) possesses the following conservative property $[3,4]$ :

$$
E(t)=\|u\|_{L_{2}}^{2}+\left\|u_{x x}\right\|_{L_{2}}^{2}=E(0) .
$$

When $-x_{L} \gg 0, x_{R} \gg 0$, the initial-boundary value problem (1)-(3) and the Cauchy problem (1) are consistent, so that the boundary conditions (3) are reasonable.

It is known that the conservative scheme is better than the nonconservative ones. The nonconservative scheme may 
easily show nonlinear blow-up. A lot of numerical experiments show that the conservative scheme can possess some invariant properties of the original differential equation [718]. The conservative scheme is more suitable for long-time calculations. In [18] Pan and Zhang said “... in some areas, the ability to preserve some invariant properties of the original differential equation is a criterion to judge the success of a numerical simulation".

The rest of this paper is organized as follows. In Section 2, we propose a Crank-Nicolson implicit nonlinear finite difference scheme for the generalized Rosenau-KdV equation and discuss the property of its solution. In Section 3, we prove that the finite difference scheme is of second order convergence. Finally, some numerical tests are given in Section 4 to verify our theoretical analysis.

\section{Finite Difference Scheme and Its Property}

Let $h=\left(x_{R}-x_{L}\right) / J$ and let $\tau$ be the uniform step size in the spatial and temporal direction, respectively. Denote $x_{j}=$ $x_{L}+j h(j=-1,0,1,2, \ldots, J, J+1), t_{n}=n \tau(n=$ $0,1,2, \ldots, N, N=[T / \tau]), u_{j}^{n} \approx u\left(x_{j}, t_{n}\right)$, and $Z_{h}^{0}=\{u=$ $\left.\left(u_{j}\right) \mid u_{-1}=u_{0}=u_{J}=u_{J+1}=0, j=-1,0,1,2, \ldots, J, J+1\right\}$. Throughout this paper, we denote $C$ as a generic positive constant independent of $h$ and $\tau$, which may have different values in different occurrences. We introduce the following notations:

$$
\begin{gathered}
\left(u_{j}^{n}\right)_{x}=\frac{u_{j+1}^{n}-u_{j}^{n}}{h}, \quad\left(u_{j}^{n}\right)_{\bar{x}}=\frac{u_{j}^{n}-u_{j-1}^{n}}{h}, \\
\left(u_{j}^{n}\right)_{\widehat{x}}=\frac{u_{j+1}^{n}-u_{j-1}^{n}}{2 h}, \quad\left(u_{j}^{n}\right)_{t}=\frac{u_{j}^{n+1}-u_{j}^{n}}{\tau}, \\
u_{j}^{n+(1 / 2)}=\frac{u_{j}^{n+1}+u_{j}^{n}}{2}, \\
\left\langle u^{n}, v^{n}\right\rangle=h \sum_{j=1}^{J-1} u_{j}^{n} v_{j}^{n}, \\
\left\|u^{n}\right\|^{2}=\left\langle u^{n}, u^{n}\right\rangle, \quad\left\|u^{n}\right\|_{\infty}=\max _{1 \leq j \leq J-1}\left\|u_{j}^{n}\right\| .
\end{gathered}
$$

We propose a conservative Crank-Nicolson finite difference scheme for the solution of (1)-(3):

$$
\begin{gathered}
\left(u_{j}^{n}\right)_{t}+\left(u_{j}^{n}\right)_{x x \bar{x} x t}+\left(u_{j}^{n+(1 / 2)}\right)_{\widehat{x}}+\left(u_{j}^{n+(1 / 2)}\right)_{x \bar{x} \hat{x}} \\
+\frac{p}{p+1}\left(u_{j}^{n+(1 / 2)}\right)^{p-1}\left(u_{j}^{n+(1 / 2)}\right)_{\widehat{x}}+\left[\left(u_{j}^{n+(1 / 2)}\right)^{p}\right]_{\widehat{x}} \\
j=1,2,3, \ldots, J-1 ; \quad n=1,2,3, \ldots, N-1, \\
u_{j}^{0}=u_{0}\left(x_{j}\right), \quad j=0,1,2,3, \ldots, J, \\
\left(u_{0}^{n}\right)_{\widehat{x}}=\left(u_{J}^{n}\right)_{\widehat{x}}=0 \quad\left(u_{0}^{n}\right)_{x \bar{x}}=\left(u_{J}^{n}\right)_{x \bar{x}}=0, \\
u^{n} \in Z_{h}^{0}, \quad n=1,2,3, \ldots, N .
\end{gathered}
$$

From the boundary conditions (3), we know that (10) is reasonable.
Lemma 1. It follows from summation by parts that, for any two mesh functions $u, v \in Z_{h}^{0}$,

$$
\begin{gathered}
\left\langle u_{x}, v\right\rangle=-\left\langle u, v_{x}\right\rangle, \quad\left\langle u_{\hat{x}}, v\right\rangle=-\left\langle u, v_{\hat{x}}\right\rangle, \\
\left\langle u_{x \bar{x}}, v\right\rangle=-\left\langle u_{x}, v_{x}\right\rangle .
\end{gathered}
$$

Then one has

$$
\left\langle u_{x \bar{x}}, u\right\rangle=-\left\langle u_{x}, u_{x}\right\rangle=-\left\|u_{x}\right\|^{2} .
$$

Furthermore, if $\left(u_{0}^{n}\right)_{x \bar{x}}=\left(u_{J}^{n}\right)_{x \bar{x}}=0$, then

$$
\left\langle u_{x x \overline{x x}}, u\right\rangle=\left\|u_{x x}\right\|^{2} .
$$

To show the existence of the solution for (7)-(10), the following Brouwer fixed point theorem should be introduced. For the proof, see [19].

Lemma 2. Let $H$ be a finite dimensional inner product space, let $\|\cdot\|$ be the associated norm, and let $g: H \rightarrow H$ be continuous. Assume, moreover, that there exists an $\alpha>0$, for all $x \in H$ and $\|x\|=\alpha,\langle g(x), x\rangle>0$. Then there exists $x^{*} \in H$ such that $g\left(x^{*}\right)=0$ and $\left\|x^{*}\right\| \leq \alpha$.

Then one has the following theorem.

Theorem 3. There exists $u^{n} \in Z_{h}^{0}$ which satisfies the difference scheme (7)-(10) $(1 \leq n \leq N)$.

Proof. In order to prove the theorem by the mathematical induction, we assume that $u^{0}, u^{1}, \ldots, u^{n}$ which satisfy (7)(10) exist for $n \leq N-1$. Next prove that there also exists $u^{n+1}$ which satisfies (7)-(10).

We define $g$ on $Z_{h}^{0}$ as follows:

$$
\begin{aligned}
g(v)= & 2 v-2 u^{n}+2 v_{x x \overline{x x}}-2 u_{x x \overline{x x}}^{n}+\tau v_{\widehat{x}} \\
& +\tau v_{x \bar{x} \bar{x}}+\frac{\tau p}{1+p}\left[v^{p-1} v_{\widehat{x}}+\left(v^{p}\right)_{\hat{x}}\right] .
\end{aligned}
$$

Taking an inner product of (14) with $v$ and considering

$$
\left\langle v_{\widehat{x}}, v\right\rangle=0, \quad\left\langle v_{x \bar{x} \hat{x}}, v\right\rangle=0, \quad\left\langle v^{p-1} v_{\widehat{x}}+\left(v^{p}\right)_{\widehat{x}}, v\right\rangle=0,
$$

we have

$$
\begin{aligned}
\langle g(v), v\rangle= & 2\|v\|^{2}-2\left\langle u^{n}, v\right\rangle+2\left\|v_{x x}\right\|^{2}-2\left\langle u_{x x}^{n}, v_{x x}\right\rangle \\
\geq & 2\|v\|^{2}-2\left\|u^{n}\right\| \cdot\|v\|+2\left\|v_{x x}\right\|^{2} \\
& -2\left\|u_{x x}^{n}\right\| \cdot\left\|v_{x x}\right\| \\
\geq & 2\|v\|^{2}-\left(\left\|u^{n}\right\|^{2}+\|v\|^{2}\right)+2\left\|v_{x x}\right\|^{2} \\
& -\left(\left\|u_{x x}^{n}\right\|^{2}+\left\|v_{x x}\right\|^{2}\right) \\
\geq & \|v\|^{2}-\left(\left\|u^{n}\right\|^{2}+\left\|u_{x x}^{n}\right\|^{2}\right)+\left\|v_{x x}\right\|^{2} \\
\geq & \|v\|^{2}-\left(\left\|u^{n}\right\|^{2}+\left\|u_{x x}^{n}\right\|^{2}\right) .
\end{aligned}
$$


Hence, it is obvious that $\langle g(v), v\rangle>0$ for all $\forall v \in Z_{h}^{0}$ with $\|v\|^{2}=\left\|u^{n}\right\|^{2}+\left\|u_{x x}^{n}\right\|^{2}+1$. It follows from Lemma 2 that there exists $v^{*} \in Z_{h}^{0}$ such that $g\left(v^{*}\right)=0$. Let $u^{n+1}=2 v^{*}-u^{n}$; then $u^{n+1}$ satisfies (7).

The difference scheme (7)-(10) simulates the conservation property of the problem (1)-(3) as follows.

Theorem 4. Suppose that $u_{0} \in H_{0}^{2}\left[x_{L}, x_{R}\right]$, then the difference scheme (7)-(10) is conservative:

$$
E^{n}=\left\|u^{n}\right\|^{2}+\left\|u_{x x}^{n}\right\|^{2}=E^{n-1}=\cdots=E^{0} .
$$

Proof. Taking an inner product of (7) with $2 u^{n+(1 / 2)}$ (i.e., $u^{n+1}+u^{n}$ ), according to the boundary condition (10) and Lemma 1 , we obtain

$$
\begin{aligned}
& \frac{1}{\tau}\left(\left\|u^{n+1}\right\|^{2}-\left\|u^{n}\right\|^{2}\right)+\frac{1}{\tau}\left(\left\|u_{x x}^{n+1}\right\|^{2}-\left\|u_{x x}^{n}\right\|^{2}\right) \\
& \quad+2\left\langle u_{\widehat{x}}^{n+(1 / 2)}, u^{n+(1 / 2)}\right\rangle+2\left\langle u_{x \bar{x} \hat{x}}^{n+(1 / 2)}, u^{n+(1 / 2)}\right\rangle \\
& \quad+2\left\langle\varphi\left(u^{n+(1 / 2)}\right), u^{n+(1 / 2)}\right\rangle=0,
\end{aligned}
$$

where $\varphi\left(u_{j}^{n+(1 / 2)}\right)=(p /(p+1))\left[\left(u_{j}^{n+(1 / 2)}\right)^{p-1} \cdot\left(u_{j}^{n+(1 / 2)}\right)_{\widehat{x}}+\right.$ $\left.\left(\left(u_{j}^{n+(1 / 2)}\right)^{p}\right)_{\widehat{x}}\right]$.

From

$$
\begin{gathered}
\left\langle u_{\widehat{x}}^{n+(1 / 2)}, u^{n+(1 / 2)}\right\rangle=0, \\
\left\langle u_{x \bar{x} \widehat{x}}^{n+(1 / 2)}, u^{n+(1 / 2)}\right\rangle=0, \\
\left\langle\varphi\left(u^{n+(1 / 2)}\right), 2 u^{n+(1 / 2)}\right\rangle \\
=\frac{2 p}{p+1} h \sum_{j=1}^{J-1}\left[\left(u_{j}^{n+(1 / 2)}\right)^{p-1} \cdot\left(u_{j}^{n+(1 / 2)}\right)_{\widehat{x}}\right. \\
\left.+u_{j}^{n+(1 / 2)}+\left(\left(u_{j}^{n+(1 / 2)}\right)^{p}\right)_{\widehat{x}}\right] u_{j}^{n+(1 / 2)} \\
=\frac{p}{p+1} h \sum_{j=1}^{J-1}\left(u_{j}^{n+(1 / 2)}\right)^{p-1} \cdot\left(u_{j+1}^{n+(1 / 2)}-u_{j-1}^{n+(1 / 2)}\right) \\
+\left[\left(u_{j+1}^{n+(1 / 2)}\right)^{p}-\left(u_{j-1}^{n+(1 / 2)}\right)^{p}\right] u_{j}^{n+(1 / 2)} \\
=\frac{p}{p+1} \sum_{j=1}^{J-1}\left[\left(u_{j+1}^{n+(1 / 2)}\right)^{p-1} u_{j}^{n+(1 / 2)}+\left(u_{j}^{n+(1 / 2)}\right)^{p}\right] \\
\cdot u_{j+1}^{n+(1 / 2)}-\frac{p}{p+1} \sum_{j=1}^{J-1}\left[\left(u_{j}^{n+(1 / 2)}\right)^{p-1} u_{j-1}^{n+(1 / 2)}+\left(u_{j-1}^{n+(1 / 2)}\right)^{p}\right] \\
\cdot u_{j}^{n+(1 / 2)}=0,
\end{gathered}
$$

we have

$$
\left(\left\|u^{n+1}\right\|^{2}-\left\|u^{n}\right\|^{2}\right)+\left(\left\|u_{x x}^{n+1}\right\|^{2}-\left\|u_{x x}^{n}\right\|^{2}\right)=0 .
$$

Then (17) is gotten from (21).
In order to prove the bounded quality of the difference solution, we introduce the following lemma.

Lemma 5 (discrete Sobolev's inequality [2]). There exist two constants $C_{1}$ and $C_{2}$ such that

$$
\left\|u^{n}\right\|_{\infty} \leq C_{1}\left\|u^{n}\right\|+C_{2}\left\|u_{x}^{n}\right\| .
$$

Theorem 6. Suppose $u_{0} \in H_{0}^{2}\left[x_{L}, x_{R}\right]$; then the solution of (7)-(10) satisfies

$$
\left\|u^{n}\right\| \leq C, \quad\left\|u_{x}^{n}\right\| \leq C,
$$

which yield

$$
\left\|u^{n}\right\|_{\infty} \leq C, \quad\left\|u_{x}^{n}\right\|_{\infty} \leq C \quad(n=1,2, \ldots, N) .
$$

Proof. It follows from (17) that

$$
\left\|u^{n}\right\| \leq C, \quad\left\|u_{x x}^{n}\right\| \leq C .
$$

According to (12) and Schwarz inequality, we get

$$
\left\|u_{x}^{n}\right\|^{2} \leq\left\|u^{n}\right\| \cdot\left\|u_{x x}^{n}\right\| \leq \frac{1}{2}\left(\left\|u^{n}\right\|^{2}+\left\|u_{x x}^{n}\right\|^{2}\right) \leq C .
$$

Using Lemma 5, we have

$$
\left\|u^{n}\right\|_{\infty} \leq C, \quad\left\|u_{x}^{n}\right\|_{\infty} \leq C .
$$

Remark 7. Theorem 6 implies that the solution of difference scheme (7)-(10) is stable in the sense of norm $\|\cdot\|_{\infty}$.

\section{Convergence}

In order to prove the convergence of the difference scheme, we need to introduce the lemma as follows:

Lemma 8 (discrete Gronwall inequality [2]). Suppose $w(k)$ and $\rho(k)$ are nonnegative functions and $\rho(k)$ is nondecreasing. If $C>0$ and

$$
w(k) \leq \rho(k)+C \tau \sum_{l=0}^{k-1} w(l), \quad \forall k,
$$

then

$$
w(k) \leq \rho(k) e^{C \tau k}, \quad \forall k .
$$

Theorem 9. Suppose that $u_{0} \in H_{0}^{2}\left[x_{L}, x_{R}\right]$; then the solution $u^{n}$ of (1)-(3) satisfies

$$
\begin{aligned}
\|u\|_{L_{2}} \leq C, & \left\|u_{x}\right\|_{L_{2}} \leq C, \\
\|u\|_{L_{\infty}} & \leq C, \quad\left\|u_{x}\right\|_{L_{\infty}} \leq C .
\end{aligned}
$$

Proof. It follows from (5) that

$$
\|u\|_{L_{2}} \leq C, \quad\left\|u_{x x}\right\|_{L_{2}} \leq C .
$$


By Holder inequality and Schwarz inequality, we get

$$
\begin{aligned}
\left\|u_{x}\right\|_{L_{2}}^{2} & =\int_{x_{L}}^{x_{R}} u_{x} u_{x} d x=\left.u u_{x}\right|_{x_{L}} ^{x_{R}}-\int_{x_{L}}^{x_{R}} u u_{x x} d x \\
& =-\int_{x_{L}}^{x_{R}} u u_{x x} d x \\
& \leq\|u\|_{L_{2}} \cdot\left\|u_{x x}\right\|_{L_{2}} \leq \frac{1}{2}\left(\|u\|_{L_{2}}^{2}+\left\|u_{x x}\right\|_{L_{2}}^{2}\right),
\end{aligned}
$$

which implies

$$
\left\|u_{x}\right\|_{L_{2}} \leq C
$$

Using Sobolev inequality, we get

$$
\|u\|_{L_{\infty}} \leq C, \quad\left\|u_{x}\right\|_{L_{\infty}} \leq C .
$$

Let $v(x, t)$ be the solution of problem (1)-(3), $v_{j}^{n}=$ $v\left(x_{j}, t_{n}\right)$; then the truncation error of the difference scheme (7)-(10) is

$$
\begin{aligned}
r_{j}^{n}= & \left(v_{j}^{n}\right)_{t}+\left(v_{j}^{n}\right)_{x x \bar{x} \bar{x} t}+\left(v_{j}^{n+(1 / 2)}\right)_{x \widehat{x}} \\
& +\left(v_{j}^{n+(1 / 2)}\right)_{x \bar{x} \widehat{x}}+\varphi\left(v_{j}^{n+(1 / 2)}\right) .
\end{aligned}
$$

Making use of Taylor expansion, we know that $r_{j}^{n}=O\left(\tau^{2}+h^{2}\right)$ holds if $h, \tau \rightarrow 0$.

Theorem 10. Suppose $u_{0} \in H_{0}^{2}\left[x_{L}, x_{R}\right]$; then the solution $u^{n}$ of the difference scheme (7)-(10) converges to the solution $v(x, t)$ of the problem (1)-(3) with order $O\left(\tau^{2}+h^{2}\right)$ in norm $\|\cdot\|_{\infty}$.

Proof. Subtracting (7) from (35) and letting $e_{j}^{n}=v_{j}^{n}-u_{j}^{n}$, we have

$$
\begin{aligned}
r_{j}^{n}= & \left(e_{j}^{n}\right)_{t}+\left(e_{j}^{n}\right)_{x x \bar{x} \bar{x}}+\left(e_{j}^{n+(1 / 2)}\right)_{\widehat{x}}+\left(e_{j}^{n+(1 / 2)}\right)_{x \bar{x} \hat{x}} \\
& +\varphi\left(v_{j}^{n+(1 / 2)}\right)-\varphi\left(u_{j}^{n+(1 / 2)}\right) .
\end{aligned}
$$

Computing the inner product of (36) with $2 e^{n+(1 / 2)}$, we obtain

$$
\begin{aligned}
\left\langle r^{n}, 2 e^{n+(1 / 2)}\right\rangle= & \frac{1}{\tau}\left(\left\|e^{n+1}\right\|^{2}-\left\|e^{n}\right\|^{2}\right) \\
& +\frac{1}{\tau}\left(\left\|e_{x x}^{n+1}\right\|^{2}-\left\|e_{x x}^{n}\right\|^{2}\right)+\left\langle e_{\widehat{x}}^{n+1}, 2 e^{n+(1 / 2)}\right\rangle \\
& +\left\langle e_{x \bar{x} \hat{x}}^{n+(1 / 2)}, 2 e^{n+(1 / 2)}\right\rangle \\
& +\left\langle\varphi\left(v^{n+(1 / 2)}\right)-\varphi\left(u^{n+(1 / 2)}\right), 2 e^{n+(1 / 2)}\right\rangle .
\end{aligned}
$$

Similar to the proof of (19), we have

$$
\begin{gathered}
\left\langle e_{\widehat{x}}^{\mathrm{n}+1}, 2 e^{n+(1 / 2)}\right\rangle=0, \\
\left\langle e_{x \bar{x} \hat{x}}^{n+(1 / 2)}, 2 e^{n+(1 / 2)}\right\rangle=0 .
\end{gathered}
$$

This indicates

$$
\begin{aligned}
& \left(\left\|e^{n+1}\right\|^{2}-\left\|e^{n}\right\|^{2}\right)+\left(\left\|e_{x x}^{n+1}\right\|^{2}-\left\|e_{x x}^{n}\right\|^{2}\right) \\
& \quad=\tau\left\langle r^{n}, 2 e^{n+(1 / 2)}\right\rangle-\tau\left\langle Q_{1}+Q_{2}, 2 e^{n+1 / 2}\right\rangle,
\end{aligned}
$$

where

$$
Q_{1}=\frac{p h}{p+1} \sum_{j=1}^{J-1}\left[\left(v_{j}^{n+(1 / 2)}\right)^{p-1} \cdot\left(v_{j}^{n+(1 / 2)}\right)_{\widehat{x}}\right.
$$

$$
\left.-\left(u_{j}^{n+(1 / 2)}\right)^{p-1} \cdot\left(u_{j}^{n+(1 / 2)}\right)_{\widehat{x}}\right],
$$

$$
Q_{2}=\frac{p h}{p+1} \sum_{j=1}^{J-1}\left[\left(\left(v_{j}^{n+(1 / 2)}\right)^{p}\right)_{\widehat{x}}-\left(\left(u_{j}^{n+(1 / 2)}\right)^{p}\right)_{\widehat{x}}\right] .
$$

Noting that

$$
\begin{aligned}
& \left\langle Q_{1}, 2 e^{n+(1 / 2)}\right\rangle \\
& =-\frac{2 p}{1+p} h \sum_{j=0}^{J-1}\left[\left(v_{j}^{n+(1 / 2)}\right)^{p-1} \cdot\left(v_{j}^{n+(1 / 2)}\right)_{\widehat{x}}\right. \\
& \left.-\left(u_{j}^{n+(1 / 2)}\right)^{p-1} \cdot\left(u_{j}^{n+(1 / 2)}\right)_{\widehat{x}}\right] e_{j}^{n+(1 / 2)} \\
& =-\frac{2 p}{1+p} h \sum_{j=0}^{J-1}\left[\left(v_{j}^{n+(1 / 2)}\right)^{p-1} \cdot\left(e_{j}^{n+(1 / 2)}\right)_{\widehat{x}} e_{j}^{n+(1 / 2)}\right] \\
& \quad-\frac{2 p}{1+p} h \sum_{j=0}^{J-1}\left[\left(v_{j}^{n+(1 / 2)}\right)^{p-1}-\left(u_{j}^{n+(1 / 2)}\right)^{p-1}\right] \\
& \quad \times\left(u_{j}^{n+(1 / 2)}\right)_{\widehat{x}} e_{j}^{n+(1 / 2)} \\
& =-\frac{2 p}{1+p} h \sum_{j=0}^{J-1}\left(v_{j}^{n+(1 / 2)}\right)^{p-1} \cdot\left(e_{j}^{n+(1 / 2)}\right)_{\widehat{x}}
\end{aligned}
$$$$
\times e_{j}^{n+(1 / 2)}-\frac{2 p}{1+p} h \sum_{j=0}^{J-1} e_{j}^{n+(1 / 2)}
$$$$
\times \sum_{k=0}^{p-2}\left[\left(v_{j}^{n+(1 / 2)}\right)^{p-2-k}\left(u_{j}^{n+(1 / 2)}\right)^{k}\right]
$$$$
\times\left(u_{j}^{n+(1 / 2)}\right)_{\widehat{x}} e_{j}^{n+(1 / 2)}
$$

$$
\begin{aligned}
& \leq C h \sum_{j=0}^{J-1}\left|\left(e_{j}^{n+(1 / 2)}\right)_{\widehat{x}}\right| \cdot\left|e_{j}^{n+(1 / 2)}\right|+C h \sum_{j=0}^{J-1}\left|\left(e_{j}^{n+(1 / 2)}\right)^{2}\right| \\
& \leq C\left(\left\|e_{x}^{n+(1 / 2)}\right\|^{2}+\left\|e^{n+(1 / 2)}\right\|^{2}\right) \\
& \leq C\left(\left\|e_{x}^{n+1}\right\|^{2}+\left\|e_{x}^{n}\right\|^{2}+\left\|e^{n+1}\right\|^{2}+\left\|e^{n}\right\|^{2}\right),
\end{aligned}
$$

$$
\begin{aligned}
& \left\langle Q_{2}, 2 e^{n+(1 / 2)}\right\rangle \\
& =-\frac{2 p}{1+p} h \sum_{j=0}^{J-1}\left[\left(\left(v_{j}^{n+(1 / 2)}\right)^{p}\right)_{\widehat{x}}-\left(\left(u_{j}^{n+(1 / 2)}\right)^{p}\right)_{\widehat{x}}\right] e_{j}^{n+(1 / 2)}
\end{aligned}
$$




$$
\begin{aligned}
= & -\frac{2 p}{1+p} h \sum_{j=0}^{J-1}\left[\left(v_{j}^{n+(1 / 2)}\right)^{p}-\left(u_{j}^{n+(1 / 2)}\right)^{p}\right]\left(e_{j}^{n+(1 / 2)}\right)_{\widehat{x}} \\
= & \frac{2 p}{1+p} h \sum_{j=0}^{J-1}\left[\sum_{k=0}^{p-1}\left(v_{j}^{n+(1 / 2)}\right)^{p-1-k}\left(u_{j}^{n+(1 / 2)}\right)^{k}\right] \\
& \times\left(u_{j}^{n+(1 / 2)}\right)_{\widehat{x}} e_{j}^{n+(1 / 2)} \\
\leq & C\left(\left\|e_{x}^{n+(1 / 2)}\right\|^{2}+\left\|e^{n+(1 / 2)}\right\|^{2}\right) \\
\leq & C\left(\left\|e_{x}^{n+1}\right\|^{2}+\left\|e_{x}^{n}\right\|^{2}+\left\|e^{n+1}\right\|^{2}+\left\|e^{n}\right\|^{2}\right)
\end{aligned}
$$

and with

$$
\begin{aligned}
\left\langle r^{n}, 2 e^{n+(1 / 2)}\right\rangle & =\left\langle r^{n}, e^{n+1}+e^{n}\right\rangle \\
& \leq\left\|r^{n}\right\|^{2}+\frac{1}{2}\left(\left\|e^{n+1}\right\|^{2}+\left\|e^{n}\right\|^{2}\right),
\end{aligned}
$$

we have

$$
\begin{aligned}
& \left(\left\|e^{n+1}\right\|^{2}-\left\|e^{n}\right\|^{2}\right)+\left(\left\|e_{x x}^{n+1}\right\|^{2}-\left\|e_{x x}^{n}\right\|^{2}\right) \\
& \leq C \tau\left(\left\|e^{n+1}\right\|^{2}+\left\|e^{n}\right\|^{2}+\left\|e_{x}^{n+1}\right\|^{2}+\left\|e_{x}^{n}\right\|^{2}\right)+\tau\left\|r^{n}\right\|^{2} .
\end{aligned}
$$

Similar to the proof of Theorem 6, we have

$$
\begin{aligned}
\left\|e_{x}^{n+1}\right\|^{2} & \leq \frac{1}{2}\left(\left\|e^{n+1}\right\|^{2}+\left\|e_{x x}^{n+1}\right\|^{2}\right) ; \\
\left\|e_{x}^{n}\right\|^{2} & \leq \frac{1}{2}\left(\left\|e^{n}\right\|^{2}+\left\|e_{x x}^{n}\right\|^{2}\right) .
\end{aligned}
$$

This yields

$$
\begin{aligned}
& \left\|e^{n+1}\right\|^{2}+\left\|e_{x x}^{n+1}\right\|^{2}-\left(\left\|e^{n}\right\|^{2}+\left\|e_{x x}^{n}\right\|^{2}\right) \\
& \leq C \tau\left(\left\|e^{n+1}\right\|^{2}+\left\|e^{n}\right\|^{2}+\left\|e_{x x}^{n+1}\right\|^{2}+\left\|e_{x x}^{n}\right\|^{2}\right)+\tau\left\|r^{n}\right\|^{2} .
\end{aligned}
$$

Let $B^{n}=\left\|e^{n}\right\|^{2}+\left\|e_{x x}^{n}\right\|^{2}$. We claim that

$$
B^{n+1}-B^{n} \leq C \tau\left(B^{n+1}+B^{n}\right)+\tau\left\|r^{n}\right\|^{2},
$$

which yields

$$
(1-C \tau)\left(B^{n+1}-B^{n}\right) \leq 2 C \tau B^{n}+\tau\left\|r^{n}\right\|^{2} .
$$

If $\tau$ is sufficiently small which satisfies $1-C \tau>0$, then

$$
B^{n+1}-B^{n} \leq C \tau B^{n}+C \tau\left\|r^{n}\right\|^{2} .
$$

Summing up (48) from 0 to $n-1$, we have

$$
B^{n} \leq B^{0}+C \tau \sum_{l=0}^{n-1}\left\|r^{l}\right\|^{2}+C \tau \sum_{l=0}^{n-1} B^{l} .
$$

Since

$$
\tau \sum_{l=0}^{n-1}\left\|r^{l}\right\|^{2} \leq n \tau \max _{0 \leq l \leq n-1}\left\|r^{l}\right\|^{2} \leq T \cdot O\left(\tau^{2}+h^{2}\right)^{2}
$$

and $B^{0}=O\left(\tau^{2}+h^{2}\right)^{2}$, we obtain

$$
B^{n} \leq O\left(\tau^{2}+h^{2}\right)^{2}+C \tau \sum_{l=0}^{n-1} B^{l} .
$$

By Lemma 8 , we get

$$
B^{n} \leq O\left(\tau^{2}+h^{2}\right)^{2}
$$

which implies

$$
\left\|e^{n}\right\| \leq O\left(\tau^{2}+h^{2}\right), \quad\left\|e_{x x}^{n}\right\| \leq O\left(\tau^{2}+h^{2}\right) .
$$

From (44), we have

$$
\left\|e_{x}^{n}\right\| \leq O\left(\tau^{2}+h^{2}\right) .
$$

By Lemma 5 we get

$$
\left\|e^{n}\right\|_{\infty} \leq O\left(\tau^{2}+h^{2}\right) .
$$

Finally, we can similarly prove the results as follows.

Theorem 11. The solution $u^{n}$ of (7)-(10) is unique.

\section{Numerical Simulations}

The difference scheme (7)-(10) is a nonlinear system about $u_{j}^{n+1}$ that can be easily solved by the Newton iterative algorithm.

Let $x_{L}=-60, x_{R}=90$, and $T=40$. According to $[3,4]$, when $p=3$, the soliton solution is as follows:

$$
\begin{aligned}
u(x, t)= & \frac{1}{4} \sqrt{-15+3 \sqrt{41}} \operatorname{sech}^{2} \frac{1}{4} \\
& \times \sqrt{\frac{-5+\sqrt{41}}{2}}\left[x-\frac{1}{10}(5+\sqrt{41}) t\right],
\end{aligned}
$$

and the initial condition is

$$
u_{0}(x)=\frac{1}{4} \sqrt{-15+3 \sqrt{41}} \operatorname{sech}^{2} \frac{1}{4} \sqrt{\frac{-5+\sqrt{41}}{2}} x .
$$

When $p=5$, the soliton solution is as follows:

$$
\begin{aligned}
u(x, t)= & \sqrt[4]{\frac{4}{15}(-5+\sqrt{34})} \operatorname{sech} \frac{1}{3} \\
& \times \sqrt{-5+\sqrt{34}}\left[x-\frac{1}{10}(5+\sqrt{34}) t\right],
\end{aligned}
$$


TABLE 1: The error at various time step.

\begin{tabular}{lcccccc}
\hline & \multicolumn{2}{c}{$\tau=h=0.25$} & \multicolumn{2}{c}{$\tau=h=0.125$} & \multicolumn{2}{c}{$\tau=h=0.0625$} \\
& $p=3$ & $p=5$ & $p=3$ & $p=5$ & $p=3$ & $p=5$ \\
\hline$t=10$ & $2.53343 e-3$ & $3.43739 e-3$ & $6.35973 e-4$ & $8.64177 e-4$ & $1.59182 e-4$ & $2.16316 e-4$ \\
$t=20$ & $4.40914 e-3$ & $6.31248 e-3$ & $1.10927 e-3$ & $1.58976 e-3$ & $2.77703 e-4$ & $3.98160 e-4$ \\
$t=30$ & $6.03109 e-3$ & $9.13274 e-3$ & $1.51828 e-3$ & $2.30402 e-3$ & $3.80216 e-4$ & $5.77288 e-4$ \\
$t=40$ & $7.53941 e-3$ & $1.20204 e-2$ & $1.89987 e-3$ & $3.03743 e-3$ & $4.75848 e-4$ & $7.61418 e-4$ \\
\hline
\end{tabular}

TABLE 2: The error comparison when $t=40$.

\begin{tabular}{lcccccc}
\hline & \multicolumn{2}{c}{$\tau=h=0.25$} & \multicolumn{2}{c}{$\tau=h=0.125$} & \multicolumn{2}{c}{$\tau=h=0.0625$} \\
& $p=3$ & $p=5$ & $p=3$ & $p=5$ & $p=3 \quad$ & $p=5$ \\
\hline Scheme I & $7.53941 e-3$ & $1.20204 e-2$ & $1.89987 e-3$ & $3.03743 e-3$ & $4.75848 e-4$ & $7.61418 e-4$ \\
Scheme II & $1.34986 e-2$ & $1.79985 e-2$ & $3.42489 e-3$ & $4.56804 e-3$ & $8.59570 e-4$ & $1.14689 e-3$ \\
\hline
\end{tabular}

TABLE 3: The verification of the second convergence.

\begin{tabular}{|c|c|c|c|c|c|c|}
\hline & \multicolumn{6}{|c|}{$\left\|e^{n}(h, \tau)\right\| /\left\|e^{2 n}(h / 2, \tau / 2)\right\|$} \\
\hline & & $p=3$ & & & $p=5$ & \\
\hline & $\tau=h=0.1$ & $\tau=h=0.05$ & $\tau=h=0.025$ & $\tau=h=0.1$ & $\tau=h=0.05$ & $\tau=h=0.025$ \\
\hline$t=10$ & - & 3.98355 & 3.99527 & - & 3.97764 & 3.99497 \\
\hline$t=20$ & - & 3.97483 & 3.99443 & - & 3.97073 & 3.99275 \\
\hline$t=30$ & - & 3.97233 & 3.99319 & - & 3.96382 & 3.99112 \\
\hline$t=40$ & - & 3.96837 & 3.99261 & - & 3.95742 & 3.98917 \\
\hline
\end{tabular}

TABLE 4: Numerical simulations on conservation invariant $E^{n}$.

\begin{tabular}{lcccccr}
\hline & \multicolumn{2}{c}{$\tau=h=0.25$} & \multicolumn{2}{c}{$\tau=h=0.125$} & \multicolumn{2}{c}{$\tau=h=0.0625$} \\
& $p=3$ & $p=5$ & $p=3$ & $p=5$ & $p=3$ & $p=5$ \\
\hline$t=0$ & 1.68252899330 & 3.11067490241 & 1.68254308255 & 3.11070293879 & 1.68254661109 & 3.11070996431 \\
$t=10$ & 1.68252899329 & 3.11067490241 & 1.68254308255 & 3.11070293879 & 1.68254661108 & 3.11070996430 \\
$t=20$ & 1.68252899328 & 3.11067490240 & 1.68254308255 & 3.11070293879 & 1.68254661095 & 3.11070996426 \\
$t=30$ & 1.68252899327 & 3.11067490240 & 1.68254308255 & 3.11070293879 & 1.68254661102 & 3.11070996417 \\
$t=40$ & 1.68252899325 & 3.11067490240 & 1.68254308254 & 3.11070293879 & 1.68254661095 & 3.11070996435 \\
\hline
\end{tabular}

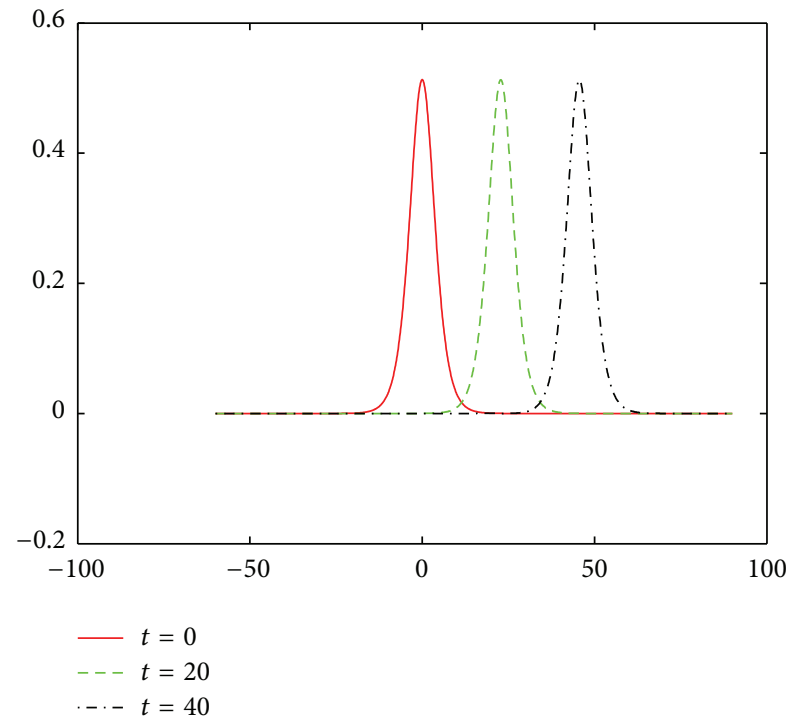

FIgURE 1: When $p=3$ and $\tau=h=0.125$, the wave graph of $u(x, t)$ at various times.

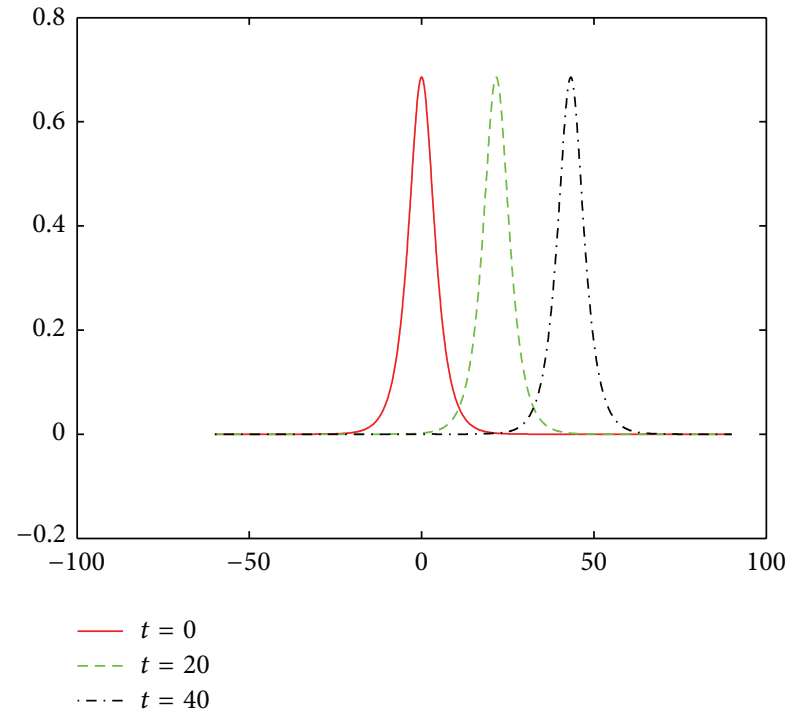

FIGURE 2: When $p=5$ and $\tau=h=0.125$, the wave graph of $u(x, t)$ at various times. 
and the initial condition is

$$
u_{0}(x)=\sqrt[4]{\frac{4}{15}(-5+\sqrt{34})} \operatorname{sech} \frac{1}{3} \sqrt{-5+\sqrt{34}} x
$$

In Table 1 we give the error at various time step. We denote the $\mathrm{C}-\mathrm{N}$ scheme in this paper as scheme I and the difference scheme in [7] as scheme II. In Table 2 we give the error comparison between scheme I and scheme II. It is easy to see that the calculation results of scheme I are slightly better than scheme II. Using the method in [20, 21], we verified the second convergence of the difference scheme in Table 3. Numerical simulations on the conservation invariant $E^{n}$ are given in Table 4.

The wave graph comparison of $u(x, t)$ at various times is given in Figures 1 and 2 when $p=3$ and $p=5$.

\section{Conclusions}

In this paper, we propose a conservative Crank-Nicolson finite difference scheme for the initial-boundary value problem of the generalized Rosenau-KdV equation. The twolevel finite difference scheme is of second order convergence and unconditionally stable, which can start by itself. From Table 2 we conclude that the C-N scheme is more efficient than scheme II in [7]. From Table 3 we conclude that the $\mathrm{C}-\mathrm{N}$ scheme is of second order convergence obviously. Numerical simulations on the conservation invariant $E^{n}$ are given in Table 4 . Figures 1 and 2 show that the height of the wave graph at different time is almost identical. Table 4 and Figures 1 and 2 imply that the finite difference scheme is conservative and efficient.

\section{Conflict of Interests}

The authors declare that there is no conflict of interests regarding the publication of this paper.

\section{Acknowledgments}

The work was supported by NSFC (61170309) and Chinese Universities Specialized Research Fund for the Doctoral Program (20110185110020).

\section{References}

[1] J.-M. Zuo, "Solitons and periodic solutions for the Rosenau$\mathrm{KdV}$ and Rosenau-Kawahara equations," Applied Mathematics and Computation, vol. 215, no. 2, pp. 835-840, 2009.

[2] J. Hu, Y. Xu, and B. Hu, "Conservative linear difference scheme for Rosenau-KdV equation," Advances in Mathematical Physics, vol. 2013, Article ID 423718, 7 pages, 2013.

[3] A. Esfahani, "Solitary wave solutions for generalized RosenauKdV equation," Communications in Theoretical Physics, vol. 55, no. 3, pp. 396-398, 2011.

[4] P. Razborova, H. Triki, and A. Biswas, "Perturbation of dispersive shallow water waves," Ocean Engineering, vol. 63, pp. 1-7, 2013.

[5] G. Ebadi, A. Mojaver, H. Triki, A. Yildirim, and A. Biswas, "Topological solitons and other solutions of the Rosenau-KdV equation with power law nonlinearity," Romanian Journal of Physics, vol. 58, no. 1-2, pp. 3-14, 2013.

[6] A. Saha, "Topological 1-soliton solutions for the Generalized Rosenau-Kdv equation," Fundamental Journal of Mathematical Physics, vol. 2, no. 1, pp. 19-23, 2012.

[7] M. Zheng and J. Zhou, "An average linear difference scheme for the generalized Rosenau-KdV equation," Journal of Applied Mathematics, vol. 2014, Article ID 202793, 9 pages, 2014.

[8] S. Li and L. Vu-Quoc, "Finite difference calculus invariant structure of a class of algorithms for the nonlinear KleinGordon equation," SIAM Journal on Numerical Analysis, vol. 32, no. 6, pp. 1839-1875, 1995.

[9] Q. Chang, E. Jia, and W. Sun, "Difference schemes for solving the generalized nonlinear Schrödinger equation," Journal of Computational Physics, vol. 148, no. 2, pp. 397-415, 1999.

[10] T.-C. Wang and L.-M. Zhang, "Analysis of some new conservative schemes for nonlinear Schrödinger equation with wave operator," Applied Mathematics and Computation, vol. 182, no. 2, pp. 1780-1794, 2006.

[11] T. Wang, B. Guo, and L. Zhang, "New conservative difference schemes for a coupled nonlinear Schrödinger system," Applied Mathematics and Computation, vol. 217, no. 4, pp. 1604-1619, 2010.

[12] L. Zhang, "A finite difference scheme for generalized regularized long-wave equation," Applied Mathematics and Computation, vol. 168, no. 2, pp. 962-972, 2005.

[13] Z. Fei and L. Vázquez, "Two energy conserving numerical schemes for the sine-Gordon equation," Applied Mathematics and Computation, vol. 45, no. 1, pp. 17-30, 1991.

[14] Y. S. Wong, Q. Chang, and L. Gong, "An initial-boundary value problem of a nonlinear Klein-Gordon equation," Applied Mathematics and Computation, vol. 84, no. 1, pp. 77-93, 1997.

[15] Q. S. Chang, B. L. Guo, and H. Jiang, "Finite difference method for generalized Zakharov equations," Mathematics of Computation, vol. 64, no. 210, pp. 537-553, 1995.

[16] J. S. Hu and K. L. Zheng, "Two conservative difference schemes for the generalized Rosenau equation," Boundary Value Problems, vol. 2010, Article ID 543503, 18 pages, 2010.

[17] X. Pan and L. Zhang, "On the convergence of a conservative numerical scheme for the usual Rosenau-RLW equation," Applied Mathematical Modelling. Simulation and Computation for Engineering and Environmental Systems, vol. 36, no. 8, pp. 3371-3378, 2012.

[18] X. Pan and L. Zhang, "Numerical simulation for general Rosenau-RLW equation: an average linearized conservative scheme," Mathematical Problems in Engineering, vol. 2012, Article ID 517818, 15 pages, 2012.

[19] F. E. Browder, "Existence and uniqueness theorems for solutions of nonlinear boundary value problems," in Proceedings of the Symposia in Applied Mathematics, vol. 17, pp. 24-49, 1965.

[20] T. Wang and B. Guo, "A robust semi-explicit difference scheme for the Kuramoto-Tsuzuki equation," Journal of Computational and Applied Mathematics, vol. 233, no. 4, pp. 878-888, 2009.

[21] J. Hu, B. Hu, and Y. Xu, "C-N difference schemes for dissipative symmetric regularized long wave equations with damping term," Mathematical Problems in Engineering, vol. 2011, Article ID 651642, 16 pages, 2011. 


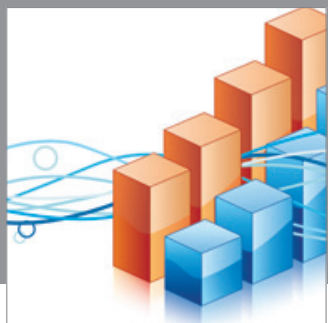

Advances in

Operations Research

mansans

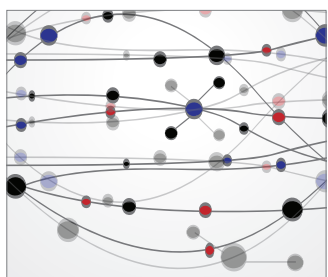

The Scientific World Journal
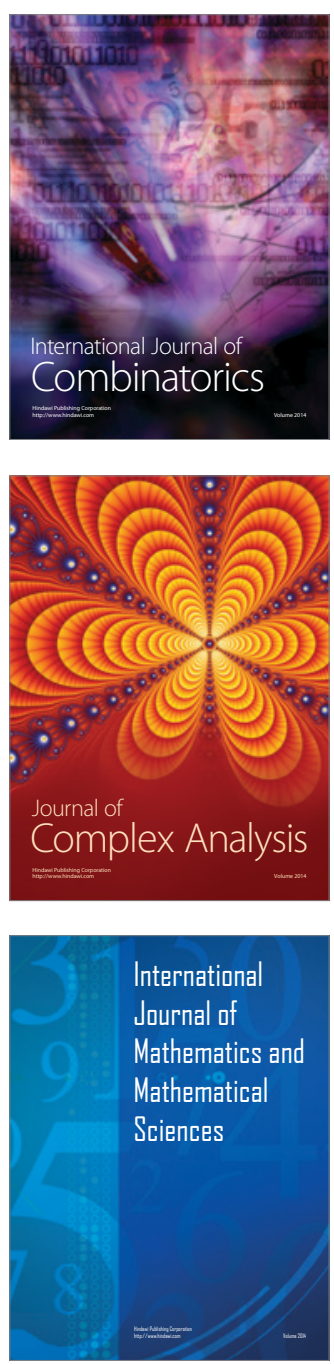
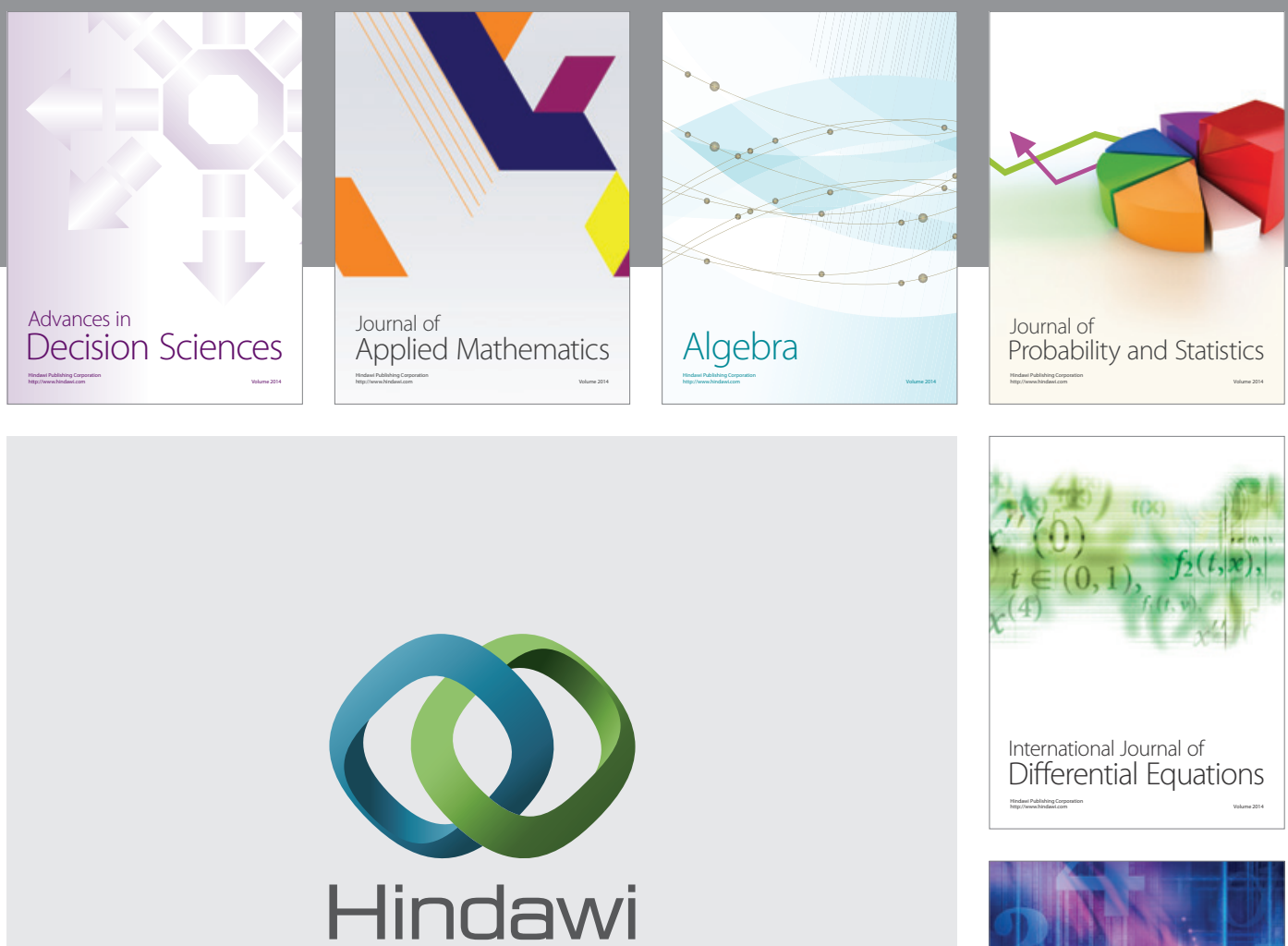

Submit your manuscripts at http://www.hindawi.com
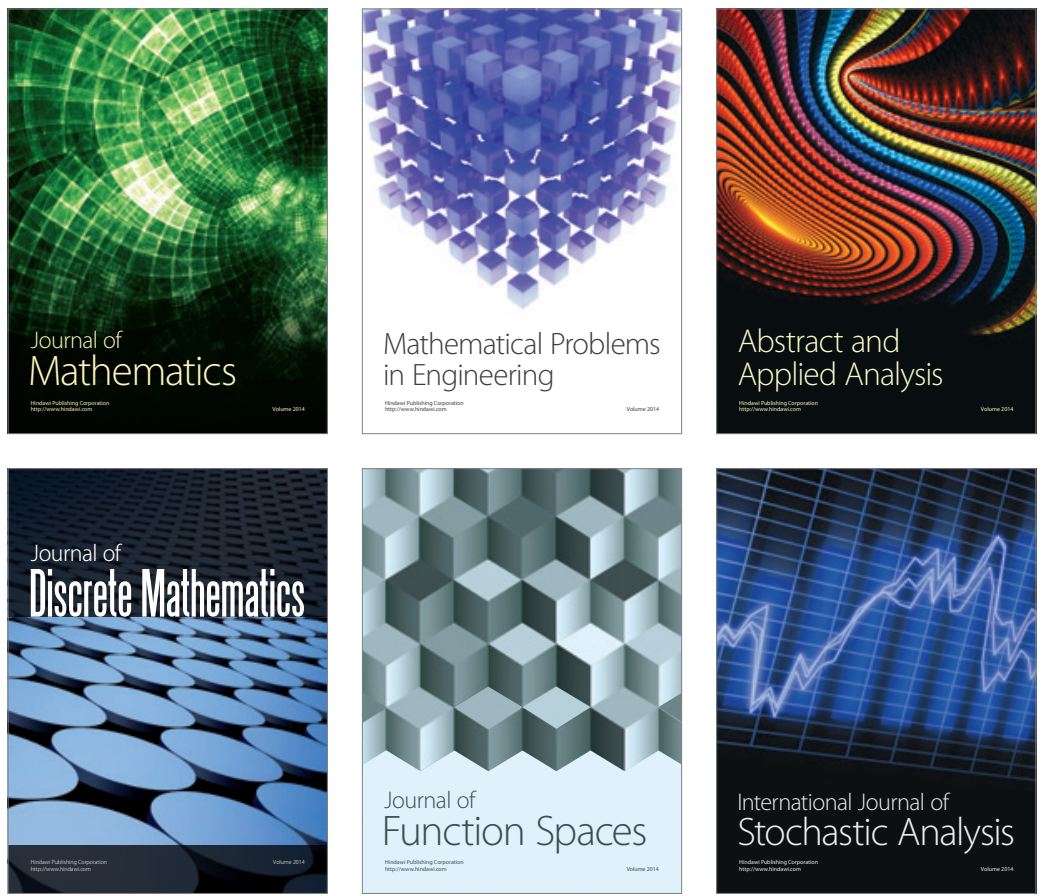

Journal of

Function Spaces

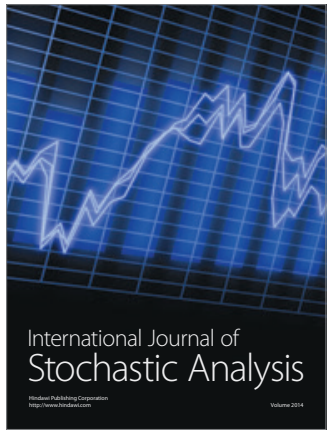

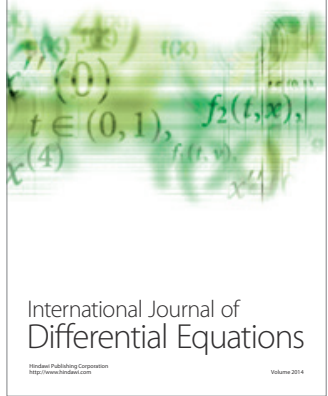
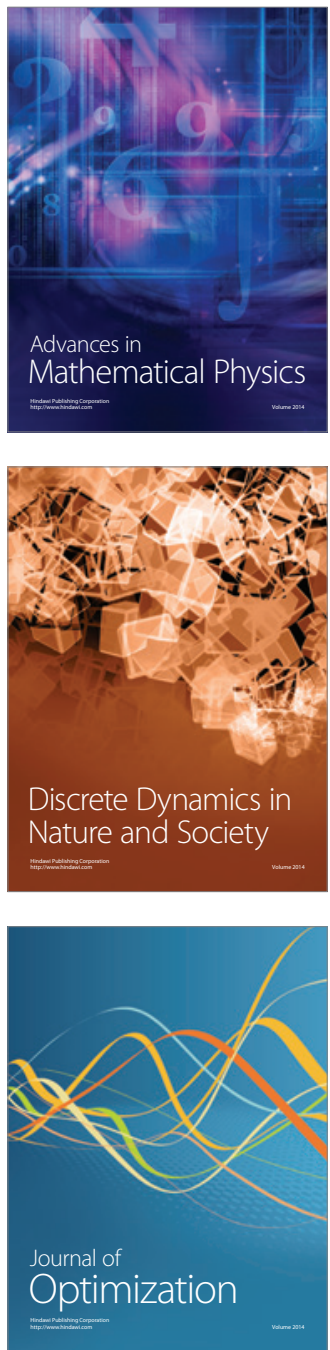